\title{
Environmental Monitoring Using Image Analysis
}

\author{
Alexey Anufriev*, Heikki Kälviäinen* and Arto Kaarna** \\ *Laboratory of Information Processing \\ **Laboratory of Telecommunications \\ Department of Information Technology \\ Lappeenranta University of Technology \\ P.O. Box 20, FIN-53851 Lappeenranta, Finland \\ Heikki.Kalviainen@lut.fi, Arto.Kaarna@lut.fi
}

\begin{abstract}
Environmental monitoring is an important task while estimating for example the influence of human activities in the nature. In this paper image analysis methods for performing these monitoring tasks are presented. We focus on the analyses of vegetation changes in lake water areas. Aerial photos taken in years 1996 and 1999 are used for the environmental monitoring of the largest Finnish lake, called Lake Saimaa. The first image analysis step is a geometrical correction that maps the analyzed images to the same coordinate system and to the same scale. The second step is to match the corresponding local regions. The third step is to find changes in vegetation. To detect vegetation we apply different classification approaches, including supervised and unsupervised classification methods. Experiments with different images, influenced by illumination, weather conditions, photographing angles, gave promising results.
\end{abstract}

\section{Introduction}

Environmental monitoring is an important task. It is important to assess the current state of the environment, to observe changes in environment caused by human activities, and try to find out relations between them. Using the feedback of such relations we can control human actions to avoid global changes in the environment. Image analysis and remote sensing [2][5][8][9] offers methods for automatic environmental monitoring.

In this study, we focus on the analyses of vegetation changes in lake water areas. Aerial photos are used for the environmental monitoring of the largest Finnish lake, called Lake Saimaa. Water vegetation was examined in 1996 and 1999. Changes in vegetation dynamics and biomass spread on the lakes are interesting factors. Conventionally this monitoring is performed by samples from water and visually interpreting airborne photographs. This is obviously time consuming and laborious since this is done manually and through laboratory experiments. The goal of this work is to compare air photographs using image analysis methods. The motivation is to build a image processing tool that could help an expert in the monitoring task. Thus, in this paper we try to solve the environmental monitoring task using remotely sensed data and digital image analysis methods. In [7], there are several approaches for land 
cover change detection, but not for water areas. The authors has reported related work in $[3][4]$.

The paper is organized as follows: Methods are described in Section 2. Experiments are shown in Section 3, and in Section 4 conclusions are given.

\section{Image Analysis for Change Detection}

\subsection{Tool for Change Detection}

The objective of this study is to build an image processing tool for the change detection of vegetation in water areas (Fig. 1). There are two images from the same region as input. The latter one is geometrically corrected and the corresponding areas are cropped. Then the vegetation change is detected in the cropped areas by three steps: (a) Color correction: the elimination of surface illumination changes; (b) Segmentation: the extraction of vegetation and water (two classes); (c) Classification: the comparisons of the class distributions.

The lake area under consideration was photographed from an airplane in 1996 and in 1999 [6]. Digital images from one of the corresponding regions are shown in Fig 2. The quality of the photographs, in terms of spatial and color accuracy, is good, but the pictures were not originally taken in a manner suited for computerized analysis. The pictures have been taken from different heights and from different angles. This produces requirements for the image analysis and an additional geometrical transformation is needed to map the regions being analyzed to the same coordinate system and to the same scale. Moreover, weather conditions can change from sunny to cloudy causing reflections on water surface, or to windy causing waves. These matters should be considered in image matching and classification.

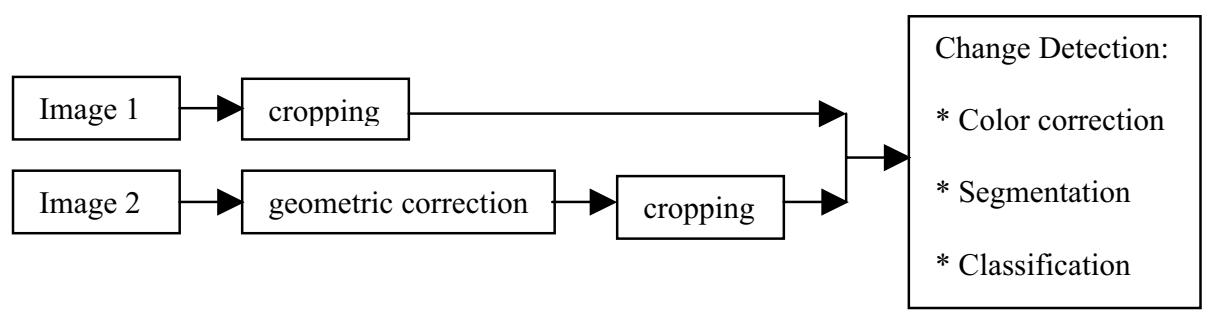

Figure 1. Image processing for change detection.

\subsection{Image Matching}

Distortions caused by the different angles of viewing must be rectified. It is necessary to ensure that the imaging distortion does not affect the estimation of the vegetated 
parts of the lake. For this purpose we use a combination of a warping algorithm with assigning pixel values to an output matrix after the transformation. The assigning is performed by resampling algorithms. Thus, in warping algorithms there are two main steps: (i) transformation and (ii) resampling. The first image is called a base image (Fig. 2.a) and the second image is called an observed image (Fig. 2.b). A warped image is computed from the observed image and it should correspond to the base image.

In the warping algorithm ground control points from the two images are needed. These points are corresponding to the same geographical locations.

The first warping method is Rotation Scaling Translation (RST) [1]. This approach uses the following affine transformation

$$
\left[\begin{array}{l}
x \\
y
\end{array}\right]=\left[\begin{array}{l}
a_{1} \\
b_{1}
\end{array}\right]+\left[\begin{array}{l}
a_{2} \\
b_{2}
\end{array}\right] \cdot X+\left[\begin{array}{l}
a_{3} \\
b_{3}
\end{array}\right] \cdot Y
$$

where $x$ and $y$ are the coordinates of a warped image, $X$ and $Y$ are the coordinates of the observed image, and $a$ and $b$ are warping coefficients.

The affine transformation can perform scaling, rotation, translation, or any combination of these. The mapping may be specified by at least three points, and a grid of triangles can be used to cover a larger area of interest. The warping coefficients are determined by solving a system of 6 linear equations with three ground control points.

Another warping method to be considered is a polynomial warping. The approach allows also shearing. The transformation function is described by the polynomial function [10]

$$
(x, y)=\left(\sum_{p}^{n} \sum_{q}^{n} a_{p q} X^{p} Y^{q}, \sum_{p}^{n} \sum_{q}^{n} b_{p q} X^{p} Y^{q}\right)
$$

where $x$ and $y$ are the coordinates of a warped image, $X$ and $Y$ are the coordinates of the observed image, and $a$ and $b$ are warping coefficients.

After producing the transformation, the resampling is used to determine new pixel values according to the transformation. Three resampling methods are used. The nearest neighbor (NN) approach uses the following scheme: the value of a pixel in the warped image is assigned equal to the value of the nearest pixel in the observed image, disregarding the slight offset. The bilinear interpolation uses the gray-levels of the four nearest pixels to determine the pixel value. Smoother results can be obtained by the cubic convolution interpolation where the weighted average of the nearest 16 pixels are used.

\subsection{Classification}

Another important part of the detection is classification. After the geometric correction the corresponding vegetated regions can be chosen in the pictures of two different years, and then apply a segmentation method to the chosen areas in order to separate vegetation and water areas. Finally, the change of vegetation is estimated. In this work, both supervised and unsupervised classification methods are used. 


\subsubsection{Supervised Classification}

Several classifiers were studied and Maximum likelihood classification [11] was found to be the most suitable one to our application area. This method is based on the well-known Bayes theorem for a posteriori probabilities

$$
P\left(\omega_{j} \mid x\right)=\frac{p\left(x \mid \omega_{j}\right) P\left(\omega_{j}\right)}{p(x)}
$$

where $p\left(x \mid \omega_{j}\right)$ is the state-conditional probability density function for $x$, i.e., the estimated probability from the training data, $P\left(\omega_{j}\right)$ is a priori probability, and $p(x)=\sum_{j=1}^{n} p\left(x \mid \omega_{j}\right) P\left(\omega_{j}\right)$. Therefore the classification rule is as follows: Decide $\omega_{j}$ if

$$
P\left(\omega_{j} \mid x\right)>P\left(\omega_{i} \mid x\right) \text { for all } j \neq i
$$

where both parts of the equation represent posteriori probabilities, giving the likelihood that the correct class is $\omega_{i}$ for $x$.

In this study a pixel is represented as a vector $x=(R, G, B)$ where $R, G$, and $B$ are the red, green, and blue pixel values. Two classes (vegetation and water pixels) exist and their probability density functions are assumed to be normally distributed.

\subsubsection{Unsupervised Classification}

In case of the unsupervised classification there is no priori information about classes, only the number of classes is known. The c-means algorithm [10] has been observed to be the most suitable to our application area. The algorithm can be presented as follows:

1. Choose the number of classes $c$.

2. Choose class centers $m_{1}, m_{2}, \ldots, m_{c}$. These are initial guesses.

3. Classify each $x$.

4. Recompute the estimates for $m_{i}$, using the result of Step 3.

5. If the $m_{i}$ are consistent, STOP; otherwise go to Step 3.

In our approach, in Step 3 each pixel is assigned to the nearest class center and in Step 4 the new cluster centers are computed as the averages of assigned pixels to each center. If the class centers have converged enough in Step 5, each pixel has been assigned to one class. 


\section{Experiments}

\subsection{Aerial Photos for Experiments}

Lake Saimaa is located in the southeastern part of Finland. Soinilansalmi is a narrow sound in Lake Saimaa [6]. From Soinilansalmi, there are aerial photos of four different places available for studying. In this project color aerial photos (RGB) for the analysis are used. The two sets of photos, from years 1996 and 1999 were provided as color photographs and then scanned and burned on CD-ROM. The 1996 photographs were taken on $6^{\text {th }}$ of August onto color negative film using a normal objective, and $24 \mathrm{~cm} \times 30 \mathrm{~cm}$ color prints were developed from the negatives. The flight altitude during the aerial photography was about $550 \mathrm{~m}$.

The 1999 aerial photographs were produced in September 1999 using a $6 \times 6$ roll film camera. The photographs were taken onto color negative film using $80 \mathrm{~mm}$ focal distance normal objective, and $20 \mathrm{~cm} \times 20 \mathrm{~cm}$ color prints were developed from the negatives.

Previous on-site measurements and assessments provided the ground truth to the experiments. According to the results [6] there have been some chances in the macrophyte water plant vegetation in Soinilansalmi water area that indicate development towards higher trophic level. Communities of emergent macrophyte species common reed (Phragmites australis) and slender tufted-sedge (Carex acuta) and floating-leaved water plants have became larger and more dense in many places. The communities of common reed and slender tufted-sedge have also become larger. Some species of water plants indicating meso- or eutrophy have increased their abundance in the research area. Chances are more obvious in the sheltered bays of the area. More detailed considerations will be published in [3].

\subsection{Results from Experiments}

For the experiments all available data sets (four pairs of images from two different years, 1996 and 1999) were taken. Currently the full set of experiments has been done for two corresponding places (Fig. 2). The two others cannot be matched correctly yet because we are still developing a better method for finding reliable ground control points.

The first phase was the geometrical correction. For the pair of images shown in Fig. 2 seven ground control points were chosen. We applied several geometric transformation methods with resampling methods mentioned in Section 2. The results of the polynomial warping and RST can been seen in Fig 3. Tests with two methods, called RST and polynomial warping, were done using three different resampling techniques: cubic convolution, NN, and bilinear interpolation. These six different experiments gave relatively similar results on the region of interest shown in Fig. 3 as 
a square, i.e., it seems that the selection of a geometric correction method is not a crucial step of the approach.

The second phase was the classification. For the supervised methods two training areas were selected for the class "vegetation" and the class "water". Results using Maximum likelihood approach and using the c-means algorithms are presented in Fig. 4. The corresponding areas are illustrated in Fig. 2 with squares. The classification between vegetation and water can been seen in Table 1 . In both experiments it can be concluded that vegetation has increased. There are some problems to exactly segment the corresponding areas as can be seen in Fig. 4.

Table 1. Classification results.

\begin{tabular}{|l|cc|cc|}
\hline Class \ Figure & Fig. 4.a & Fig. 4.b & Fig. 4.c & Fig. 4.d \\
\hline Vegetation (pixels) & 39503 & 66981 & 26102 & 39311 \\
Vegetation (\%) & 15,78 & 26,76 & 16,31 & 24,6 \\
Water (pixels) & 210787 & 183309 & 133898 & 120689 \\
Water (\%) & 84,22 & 73,24 & 83,69 & 75,4 \\
\hline
\end{tabular}

\section{Conclusions}

Vegetation dynamics monitoring on the lake using digital image analysis has been investigated. The developed method consists of geometric correction, cropping, color correction, segmentation, and classification. Based on the preliminary results of applied methods, the changes of vegetation can be assessed. The statistics of classified regions estimate the degree of changes. It has been shown that the monitoring can be automated.

Further research is needed since the current image analysis tool should cope better with various types of data. Air photographs are taken with different camera setups and viewing angles, weather conditions change (sunny, cloudy, windy, etc.), new water plants grow, and new additional objects appear on the lake.

In the geometrical correction a better method for finding reliable ground control points must be developed. Current results were compared by overlaying the base and transformed images and it was observed that the task is difficult. The RST method combined with the cubic convolution resampling method gave the best result. Moreover, the use of spectral data seems useful. Based on the vegetation spectral signatures we could classify even the types of vegetation. The most appropriate data for our purposes is the high-resolution airborne spectral images of $400 \mathrm{~nm}-1300 \mathrm{~nm}$ that includes visible and near-infrared diapasons. 


\section{Acknowledgements}

The authors would like to thank Lake Saimaa Protection Association and Kymijoki Protection Association for providing data and for valuable expert comments, especially Mr. Matti Jantunen. This study is supported by the International Master's Program in Information Technology (IMPIT) in Lappeenranta University of Technology (LUT) in Finland. Moreover, the authors are grateful to Prof. Pekka Toivanen and Mr. Janne Tuominen for useful support in the very beginning of the project.

\section{References}

1. ENVI Tutorials. Research Systems, 2001.

2. Gonzalez, R.C., Woods, R.E.: Digital Image Processing. Addison-Wesley, $2^{\text {nd }}$ edition, 2002.

3. Kaarna, A., Kälviäinen, H., Anufriev, A., Mankki, J., Malkavaara, T., Jantunen, M.: Geometric Correction and Segmentation of Images in Change Detection of Water Plants in Soinilansalmi. Accepted to Proceedings of International Geoscience and Remote Sensing Symposium, IGARSS'2003, Toulouse, France, 2003.

4. Kaarna, A., Spacil, R., Jantunen, M.: Pattern Recognition Methods as Tools of Restoration Project in Maavesi Water Area, Proceedings of International Geoscience and Remote Sensing Symposium, IGARSS'2002, Toronto, Canada, 23-27 June, 2002, vol VI, pp. 3264-3266.

5. Lillesand, T.M., Kiefer, R.W.: Remote Sensing and Image Interpretation. John Wiley and Sons, 2000.

6. Mankki, J., Malkavaara, T.: Water Plants in Soinilansalmi at Ruokolahti, Resume of the actions in 1996-1999. Kymijoki Water Protection Association, Report No. 31/2000 (in Finnish).

7. Proceedings of the International Geoscience and Remote Sensing Symposium (IGARSS 2002), Toronto, Canada, 2002.

8. Schalkoff, R.: Pattern Recognition: Statistical, Structural, and Neural Approaches. John Wiley \& Sons, Inc., 1992.

9. Richards, J.A.: Remote Sensing Digital Image Analysis. Springer-Verlag, Berlin, 1993.

10. Thomas, P.: Image Registration by Differential Evolution. Proceedings of Irish Machine Vision and Image Processing Conference, Londonderry, Northern Ireland, September 1013, 1997, pp. 221-225.

11. Tou, J.T., Gonzalez, R.C.: Pattern Recognition Principles. Addison-Wesley, 1974. 


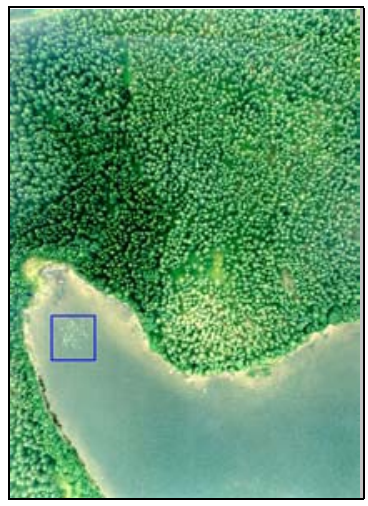

(a)

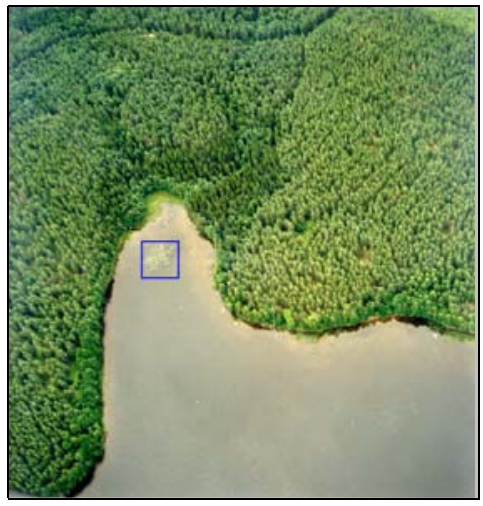

(b)

Fig. 2. Images: (a) Base image from 1996; (b) Observed image from 1999.

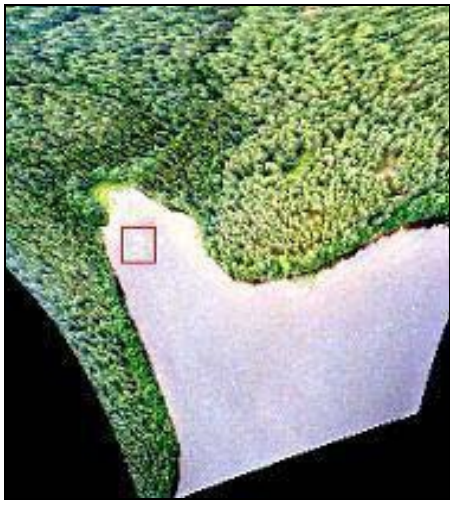

(a)

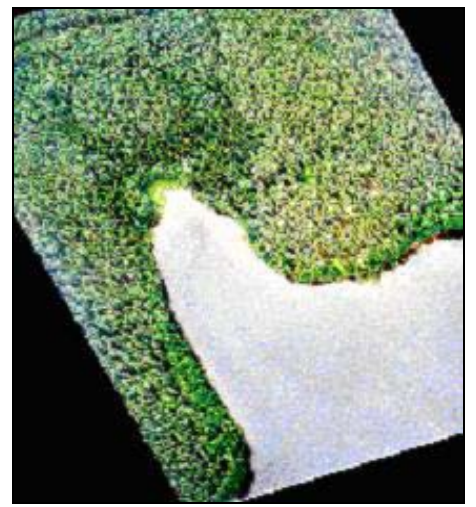

(b)

Fig 3. Geometric correction: (a) Polynomial warping with nearest neighbor resampling; (b) RST with cubic convolution.

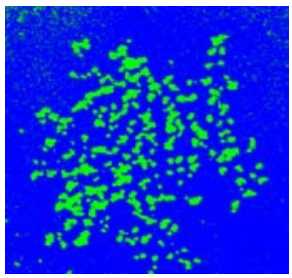

(a)

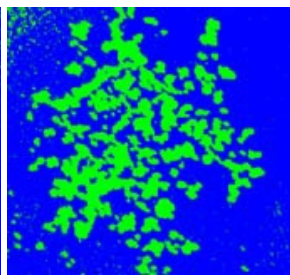

(b)

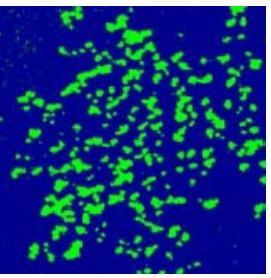

(c)

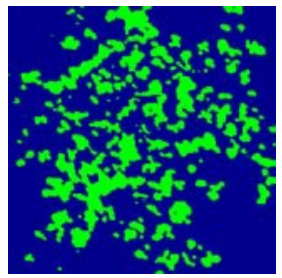

(d)

Fig. 4. Classification: Maximum likelihood approach in (a) 1996 and (b) 1999; c-means algorithm in (c) 1996 and (d) 1999. 